\title{
Identification radiographique de la position des sondes d'alimentation entérales par les infirmières de réanimation : expérience prospective bicentrique
}

\section{Radiographic identification of the appropriate position of the feeding tube by critical care nurses: a two centre prospective trial}

\author{
B. Guyon $\cdot$ Y. Martin $\cdot$ J.-D. Ricard $\cdot$ D. Coadic $\cdot$ V. David $\cdot$ P. Trouiller $\cdot$ E. Touré $\cdot$ J. Messika $\cdot$ B. Sztrymf $\cdot$ \\ le groupe DRIP-IDE
}

Reçu le 27 mars 2018; accepté le 3 mai 2018

(C) SRLF et Lavoisier SAS 2018

Il est primordial de vérifier la position des sondes d'alimentation entérale avant de commencer la nutrition entérale, car un mauvais placement dans les bronches ou la gorge peut entraîner de graves lésions pulmonaires. L'évaluation radiographique de la position par un médecin est actuellement la règle [1]. De nos jours, l'autonomie des infirmières est en augmentation [2], avec des effets bénéfiques par exemple dans le domaine de la sédation ou du contrôle glycémique [3]. Nous avons cherché à tester l'acuité des infirmières concernant la vérification radiographique de la bonne position des sondes d'alimentation, avant et après une séance ponctuelle de formation. C'était une étude prospective,

\footnotetext{
B. Guyon · D. Coadic $\cdot$ V. David · P. Trouiller · B. Sztrymf $(\bowtie)$ Service de réanimation polyvalente, CHU Antoine-Béclère, Assistance publique-Hôpitaux de Paris, université Paris-Sud, hôpital Antoine-Béclère, 157, rue de la Porte-de-Trivaux, F-92140 Clamart, France

e-mail : benjamin.sztrymf@abc.aphp.fr

Y. Martin · J.-D. Ricard · E. Touré · J. Messika

Service de réanimation médicochirurgicale,

Assistance publique-Hôpitaux de Paris,

hôpital Louis-Mourier, F-92700 Colombes, France

J.-D. Ricard · J. Messika

Institut national de la santé et de la recherche médicale, Inserm, IAME 1137, université Paris-Diderot,

F-75018 Paris, France

B. Sztrymf

Institut national de la santé et de la recherche médicale, Inserm U999, F-92060 Le Plessis-Robinson, France

le groupe DRIP-IDE

Services de réanimation des hôpitaux Antoine Béclère et Louis Mourier, Assistance Publique-Hôpitaux de Paris,

157 rue de la porte de Trivaux, F-92140 Clamart France
}

bicentrique, observationnelle, se déroulant sur huit mois selon un modèle " avant-après ». Comme dans la pratique quotidienne, une radiographie pulmonaire était réalisée après la mise en place de la sonde d'alimentation. L'infirmière en charge du patient examinait cette radiographie préalablement au médecin en charge et répondait à la question suivante : « La sonde d'alimentation est-elle dans l'estomac? » Les réponses possibles étaient « oui », « non » et « ne sait pas ». La séance de formation consistait en une présentation de diapositives de 30 minutes et portait sur les bases de l'anatomie radiographique thoracique. Elle visait à permettre l'identification de la position de la sonde d'alimentation, ce qui était ensuite testé par un ensemble de radiographies d'exercice. Les réponses du médecin ont servi de contrôle, et la capacité de l'infirmière à vérifier la position a été évaluée par le calcul de l'agrément interévaluateur par score de kappa. Le protocole a été approuvé par le comité d'éthique de la Société de réanimation de langue française. Les résultats sont exprimés en moyenne et intervalle de confiance à $95 \%$ ou pourcentage. Les comparaisons sont faites à l'aide du test de Chi2.

Deux cent trente-neuf placements de sondes d'alimentation chez 150 patients et 204 placements de sondes chez 100 patients ont été analysés respectivement avant et après la formation. Quatre-vingt-huit pour cent des patients étaient intubés. Le score global de kappa était de 0,55 $(0,36-0,74)$ avant la formation et de $0,72(0,59-0,84)$ après la formation. L'agrément passait ainsi de " moyen » à « satisfaisant » (Tableau 1) [4]. Les variations de l'agrément étaient plus prononcées au cours de la première période (Fig. 1). Un désaccord entre l'infirmière et le médecin responsable survenait respectivement dans 20 et 18 cas au cours des phases " avant " et " après » de l'étude, sans différence statistique entre les deux périodes (20/239 contre 18/204, $p=0,87)$. Dans trois cas $(1,3 \%)$ 
avant et trois cas $(1,5 \%)$ après le cours, l'infirmière a conclu à un placement correct, contrairement à l'évaluation du médecin. Il ne s'agissait dans aucun de ces cas de positions intrabronchiques des sondes, mais de placements œsophagiens ou pharyngés. Dans trois cas $(1,3 \%)$ avant et quatre cas $(1,9 \%)$ après le cours, l'infirmière a conclu à un mauvais placement de la sonde, alors que le médecin a déclaré qu'elle était bien intragastrique. Il faut également noter les situations où il n'était pas possible de répondre. Cela était toujours lié à l'absence de visualisation de la sonde sur la radiographie. Cela est survenu avant la formation 13 fois pour les infirmières et trois fois pour les médecins, et après la formation 17 fois pour les infirmières et six fois pour les médecins. Un nouveau contrôle radiographique était systématiquement entrepris dans ces situations.

Le contenu et le caractère ponctuel du cours peuvent expliquer l'évolution observée de la capacité des infirmières à conclure sur le contrôle radiographique de la sonde

Tableau 1 Interprétation du score kappa (selon Landis et Koch [4])

$<0$ : grand désaccord

0,00 à $0,20:$ accord très faible

0,21 à 0,40 : accord faible

0,41 à $0,60:$ accord moyen

0,61 à $0,80:$ accord satisfaisant

0,81 à $1,00:$ accord excellent d'alimentation. En effet, bien que les infirmières françaises en soins intensifs connaissent les bases de l'anatomie thoracique, elles ne sont pas formées pour analyser l'imagerie radiographique. Par conséquent, une seule séance d'entrâ̂nement pourrait ne pas être suffisante pour atteindre une telle compétence, et des programmes d'entraînement répétés semblent être nécessaires pour maintenir un niveau optimal et stable de capacités [5]. À l'issue de cette étude, aucun changement de pratiques n'a semblé souhaitable dans ce domaine. Différentes options sont envisageables pour améliorer l'acuité des infirmières sur ce point comme des sessions de cours répétées ou des séances de simulation. Une situation d'autonomie « partielle » pour des cas paraissant sans ambiguïté pourrait également être testée. L'autonomisation des infirmières de réanimation pour certains aspects de la prise en charge des patients a montré des gains importants. Cette autonomie professionnelle est aussi l'un des piliers de la satisfaction professionnelle des infirmières [6]. À l'heure où les autorités de tutelle envisagent de mettre en place des formations aboutissant au statut d'infirmière aux « compétences élargies » à l'image d'autres pays, il est probable que le statut des infirmières va connaître des évolutions vers encore plus d'autonomie.

Notre expérience s'inscrit dans cette optique générale et aborde de façon pragmatique un des aspects possibles de cette autonomisation qui nécessitera un cadre pédagogique et réglementaire bien défini.

Liens d'intérêts : Les auteurs déclarent ne pas avoir de lien d'intérêt.

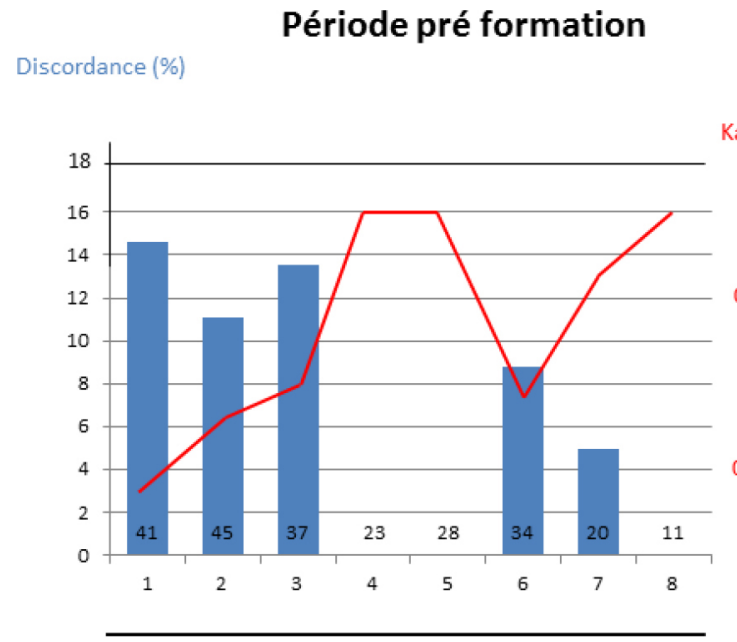

Score Kappa 0,55 (0,36-0,74)

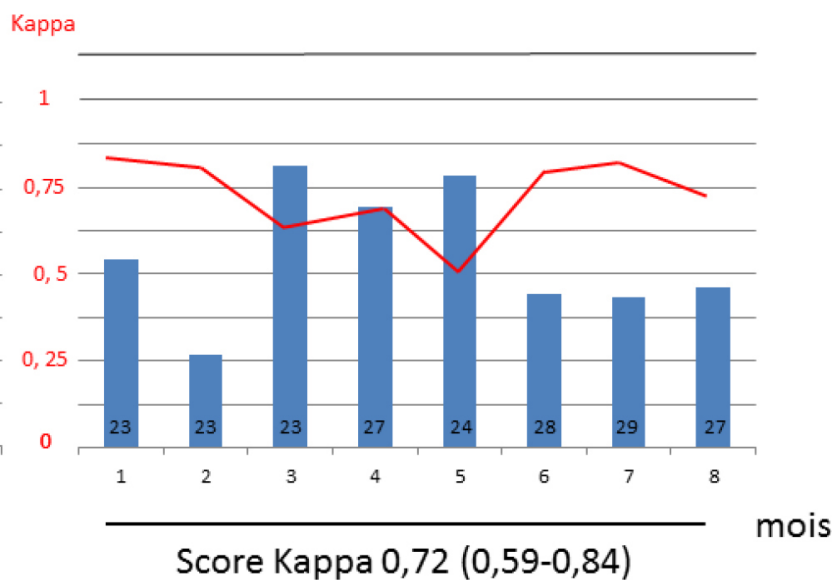

Score Kappa 0,72 (0,59-0,84)

Fig. 1 Évolution du score kappa (ligne) et du pourcentage de réponses divergentes (barres) pendant les huit mois avant et après la formation ponctuelle. Les chiffres à la base des barres indiquent le nombre de patients inclus dans le mois. Le score kappa était respectivement de $0,55(0,36-0,74)$ et de $0,72(0,59-0,84)$ avant et après la session de formation 


\section{Références}

1. Blumenstein I, Shastri YM, Stein J, (2014) Gastroenteric tube feeding: techniques, problems and solutions. World J Gastroenterol 20: $8505-8524$

2. Martinez-González NA, Djalali S, Tandjung R, Huber-Geismann F, Markun S, Wensing M, Rosemann T, (2014) Substitution of physicians by nurses in primary care: a systematic review and meta-analysis. BMC Health Serv Res 14: 214

3. Landis JR, Koch GG, (1977) The measurement of observer agreement for categorical data. Biometrics 33: 159-174
4. Dubose J, Nomoto S, Higa L, Paolim R, Teixeira P, Inaba K, Demetriades, D, Belzberg H, (2009) Nursing involvement improves compliance with tight blood glucose control in the trauma ICU: a prospective observational study. Intensive Crit Care Nurs 25: $101-107$

5. Aman M, McLaughlin K, Violato C, Rostom A, Allard JP, Coderre S, (2010) Teaching in small portions dispersed over time enhances long-term knowledge retention. Med Teach 32: $250-255$

6. Zangaro GA, Soeken KL, (2007) A meta-analysis of studies of nurses' job satisfaction. Res Nurs Health 30: 445-458 